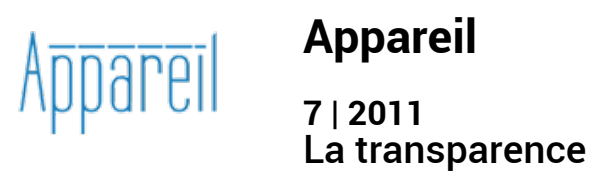

\title{
Pour un peu transparents
}

\section{Gérard Pommier}

\section{OpenEdition}

Journals

Édition électronique

URL : http://journals.openedition.org/appareil/1232

DOI : 10.4000/appareil.1232

ISSN : 2101-0714

Éditeur

MSH Paris Nord

Référence électronique

Gérard Pommier, "Pour un peu transparents », Appareil [En ligne], 7 | 2011, mis en ligne le 04 avril 2011, consulté le 30 juillet 2020. URL : http://journals.openedition.org/appareil/1232 ; DOI : https:// doi.org/10.4000/appareil.1232

Ce document a été généré automatiquement le 30 juillet 2020.

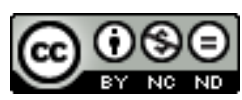

Appareil est mis à disposition selon les termes de la Licence Creative Commons Attribution - Pas d'Utilisation Commerciale - Pas de Modification 4.0 International. 


\title{
Pour un peu transparents
}

\author{
Gérard Pommier
}

1 Failli. Nous avons failli être transparents. La transparence d'origine, c'est que le corps a d'abord été désiré ailleurs, avant même sa naissance, point d'incandescence d'un désir brûlant bien avant l'apparition de la chair. En matière de transparence, qui dit mieux que ce pur néant? Nous sommes nés comme ça: presque transparents, puis, peu à peu nous sommes devenus seulement translucides, et, enfin, nous avons viré d'un seul coup à l'opaque, au fur à mesure que notre premier cri s'est prolongé. Bizarrement, c'est la sonorité de notre propre voix qui sut, en un laps de temps aussi bref que celui d'une expiration sonore, plomber un corps qui n'était pas encore notre propre, et à travers lequel on aurait pu voir. Car il faut dire que notre cri fut - le premier - ce qui nous fut propre: de sorte que ce n'est pas tant que notre corps serait devenu opaque pendant l'expiration du premier cri, c'est plutôt qu'il fut totalement décalé et tomba dans des sortes de limbes extérieures, où il demeure depuis ce temps d'une seule expiration. Depuis cet instant, le corps reste suspendu comme un sac, dans un dehors que nous ne pouvons plus appréhender que de l'extérieur, par notre propre regard ou par celui des autres, surtout lorsqu'ils nous aiment - mais aussi lorsqu'ils nous détestent. Perdre la transparence en l'espace d'un seul cri, c'est vite dit. Mais pourquoi ce corps devrait-il s'opacifier en même temps que l'action le relègue? Il devrait garder son angélisme d'origine! Or, ce n'est pas l'expérience ordinaire : très vite, nous devenons opaques à nos propres perceptions, au point qu'un enfant ne se reconnaîtra jamais dans un miroir, si quelqu'une ne le nomme pas par son nom lorsqu'il se regarde. On voit bien que le corps est rejeté dans une extériorité, qu'il passe au second plan, ou même est appendu dans une sorte de dehors inassignable (les limbes) à partir du moment où le sujet est celui de l'acte de crier, qui en quelque sorte se rejette lui-même comme objet. Un dicton populaire conseille de «ne pas jeter le bébé avec l'eau du bain ». Mais la sagesse des nations n'a pas prévu que le bébé se jette lui-même en criant : il se rejette en déployant son activité dans un registre vocal où son corps s'oublie.

2 Le cri a brisé la glace de la transparence : son choc ressemble à l'impact d'un gravillon sur un pare-brise de voiture, dont la vitre s'opacifie d'un coup. Mais cette métaphore ne rend pas compte de la cause d'un tel événement. Elle montre un résultat pour toujours laissé brut, et cherchant ensuite dans les raisonnements, les suites de pensées ou les 
calculs, le motif d'une opacité qui ne sera jamais levée par les mots - puisque ces derniers naissent à proportion de l'opacification. C'est que le pli grammatical de l'énonciation vocale - la parole qui débute avec le cri - engendre le « visible effacement de celui qui parle " pour reprendre cette formule de Foucault ${ }^{1}$ assez paradoxale. Car, comment un " effacement » peut-il être en même temps " visible »? C'est pourtant tout le problème que cherche à résoudre l'appareil psychique, à commencer par l'hallucinose des rêves, et ensuite par le recouvrement de chaque sensation par cette transparence vivante - qui est dehors comme le dehors et lui donne son âme. Le monde autour de nous est animé par notre propre transparence, qui y a trouvé refuge. Toutes les choses perçues, par exemple, sont affublées d'un genre masculin ou féminin que leur donne notre humanité obsédée par le sexe (sinon, pourquoi dirait-on «la » mer, «le» cheval, etc. ?). Notre transparence d'origine vit toujours, projetée sur le monde : elle s'anime et tremble au bout de chaque phrase, qu'elle porte jusqu'à leur terme. Nous sommes violemment poussés à penser et à parler dans l'obsession de nous rattraper. Nous parlons infiniment depuis cet exil premier du cri, pour nous rejoindre sur cet horizon (mais quelqu'un peut-il voyager jusqu'à l'horizon?) Nous traversons des paysages qui s'ouvrent aussitôt en avant, et se referment au même instant en arrière de nous.

3 Et de même, nous roulons sur les rails des mots, dans l'oubli de notre corps, dont nous n'avons de nouvelles que grâce à des succursales lointaines du cri : des mots qui se souviennent de blessures passées, s'agrafent entre eux et viennent faire symptôme plus ou moins douloureux, lieux de notre mémoire psychique bien plus que le cerveau, qui ne nous adresse jamais la parole. Le symptôme témoigne pour une forme d'opacité locale du corps, une sorte d'épaisseur sporadique en tout cas pour la conscience, puisque de son point de vue, ce symptôme est incompréhensible et demeure inscrutable, en effet. La transparence du début s'opacifie à cause d'une sorte de pliure. Tout se passe comme si la pure signification de départ se rabattait, se pliait sur des significations locales provisoires qui l'opacifiaient : la chose qui rentre ou celle qui sort de l'organisme devient le véhicule fini de l'infini, et, en même temps, le corps psychique d'origine vient se plier autour de ces significations locales : par exemple, la nourriture, ou les excréments, etc. Ce n'est pas le corps, et pourtant le seul corps accessible se replie, donc s'opacifie derrière ce qui n'est pas le corps : c'est le pli infini qu'impriment les pulsions sur une transparence première. Notre " être » s'embusque derrière ces plis des pulsions, qui instrumentent les organes. L'impossible de sa propre transparence perd ainsi le sujet depuis le départ: elle l'égare dans des sortes d'appropriations off shore, dans des colonies en mal de Mère-patrie - ces zones pulsionnelles qui externalisent le corps et seront d'ailleurs plus tard en effet les bases de repli des symptômes, ces lieux de souffrances qui assurent une improbable existence charnelle. Le symptôme est en quelque sorte l'opacification du corps qui fait retour après coup, avec retard, à partir de ce qui arrive, d'un sensible actuel qui remémore un passé refoulé. Le symptôme en ce sens, nous attend au tournant, en avant de nous, en embuscade. Et brusquement, il nous saisit sans que nous connaissions la moindre de ses raisons. Cette saisie physique est proportionnelle à l'oubli de sa cause. C'est la prise partielle d'un organe, d'une fonction, d'une zone érogène (un peu comme lorsque quelqu'un nous retient un instant par la manche). Nous n'avons de nouvelles de notre corps que sporadiquement, partiellement, et surtout après coup. C'est une érogénéité qui est comme le désir, tracté par le futur (car, contrairement à ce qui se dit, l'inconscient ne vient pas du passé, où il puise seulement sa formalisation. 
L'inconscient nous attend au futur). Il arrive aussi que nous en ayons des nouvelles directes, totales, pétrifiantes, ou brusquement la transparence se perd. C'est l'instant de la honte : la ressaisie du corps, par toutes les pulsions, qui, d'un seul coup montent au visage, à l'heure où notre nom nous fait défaut pour répondre à une situation actuelle. Prendre son nom, c'est tuer le père - passer de la honte à la culpabilité donc. En quelque sorte, la honte parle d'un "avant coup", alors que le symptôme nous attend plus loin, « après coup ".

Nous pouvons donc connaître au moins la couleur de ce qui opacifie la transparence : c'est le rouge. Peut-on en déduire quelque chose sur ce que fut la transparence d'origine? Si c'est le néant, le noir l'habille-t-elle ? Mais ce serait déjà trop dire, car les couleurs ne sont pas dans les choses, mais résultent de l'intensité de la lumière qui les traverse. Dans son De Anima, Aristote écrit que l'avènement de la couleur « existe bien dans la limite du corps, mais n'est pas du moins la limite du corps. Il faut plutôt penser que la même nature qui, extérieurement est le véhicule de la couleur, existe aussi à l'intérieur du $\operatorname{corps}^{2} »$. Un événement visible ne se réduit pas à l'évènement de sa surface, il porte en lui un bougé, plus ou moins en équilibre. Toute perception tremble un instant sur place. C'est moins une dislocalité, comme l'avance Didi-Huberman ${ }^{3}$ qu'une distemporalité, homogène si l'on veut au symptôme hystérique (à condition de considérer l'hystérie comme la normalité). Tout sujet est constamment altéré par le changement sur place que provoque l'oscillation entre l'après de la perception et l'avant de son corps reflété par cette perception, passant du diaphane à l'opaque, tout du moins à chaque instant de conscience. Voila pourquoi les perceptions peuvent nous renvoyer si loin en arrière dans notre passé: nous faire voyager, rêver dans ce tremblement de la vision, qui, selon Aristote, est un changement, mais immobile ${ }^{4}$. C'est une simultanéité contradictoire qui en rajoute sur la mise en tension douloureuse du symptôme, sur sa bisexualité, sur son bilinguisme, et qui donne son présent au corps, entre l'infantile, en effet, et le futur de la réalisation d'un désir hallucinatoire dans son principe. Cette prise brutale d'un corps vivant au présent (enfin) se produit sur le fond de sa transparence. Généralement, quand nous sommes conscients, que nous pensons et parlons, nous oublions notre corps. Mais est-ce vraiment un oubli, puisque ce corps a toujours déjà été porté disparu dans la réverbération du sensible. Toujours déjà porté disparu et rattrapé d'une seule main dans le reflet du miroir (c'est pourquoi nous sommes droitiers ou gauchers, mais de toute façon à moitié sacrifiés). Qu'est-ce qu'on repêche de ce corps qui revient du dehors : la douleur du symptôme est la plus propre, on l'a dit, mais il faut aussi lui ajouter toutes les autres formations de l'inconscient, dont certaines donnent du plaisir (comme l'orgasme).

Leibniz parle d'une sorte de nécessité du corps, une exigence qui s'imposerait à proportion de l'obscurité de notre pensée. L'esprit serait sombre, et sa nature exigerait la singularité physique : «l'exigence individuée d'avoir un corps qui nous appartient » commente Deleuze dans Le pli ${ }^{5}$. Pourtant, comment s'imposerait une telle exigence d'appropriation, sinon parce que nous commençons par perdre ce corps dont la pensée est incontestablement issue ? En fait, la conscience du corps se perd à proportion du mouvement de cette pensée, qui, en elle-même, est potentiellement consciente, et généralement claire dès qu'elle devient réflexive. Il semble donc qu'il faille renverser la proposition de Leibniz, ou plutôt lui donner un statut secondaire, succédant à une perte première, à une chute dans l'opacité. La conquête du corps, ou, dans le même mouvement transitif, la conquête de la réalité, réclame un effort constant à partir du différentiel transparence/opacité, différentiel qui partout cherche sa preuve dans les 
sensations, qui calculent à l'infini ce qui distingue une qualité d'une autre, preuve qui lui évite de sombrer à nouveau dans l'indistinction, dans un monde d'avant le départage du ciel et des eaux, du jour et de la nuit, du transparent et de l'opaque. La perception, tout du moins consciente, est toujours secondaire au différentiel interne qui la recherche avant de l'avoir trouvée (c'est-à-dire avant de la subir passivement). La recherche de qualités distinctes précède leur rencontre effective. Les différences ne sont perçues dans les choses qu'à la condition de la différence qui menace de précipiter l'obscur vers le clair dans une chute irrémédiable dans les limbes du début.

Car le corps d'origine, quant à lui toujours aussi transparent, continue de pendre dans ces limbes où il flotte sans rien attendre, éternel, suspendu comme le désir qui le nimba de son Aura angélique, celle qu'il avait juste avant le premier cri - autant dire aussi bien jamais que toujours. C'est ce désir d'avant, Autre, qui faillit faire de ce corps sa pure transparence transitive - aussitôt reléguée dans les oubliettes par l'expiration du cri. Ce corps bulle de savon flotte, à peine séparé de son extérieur par ses irisations, qui laissent seulement voir autre chose que lui : c'est une enveloppe séparée simplement par les reflets de ce qu'il n'est pas. Pourtant, ce corps flottant ne nous est pas étranger : c'est notre plus ancien intime, celui de la première seconde de la vie, plus grande que toutes les autres. La " première seconde » de la vie : quelle étrange formule ! Il n'y a pas de " première » mais seulement une " seconde » repliée sur la première qu'elle masque. La seconde toujours déjà pliée sur la transparence, c'est pour le meilleur et pour le pire! C'est l'heure de rejoindre un instant sa bulle, surplomb du temps, et de la fuir aussitôt pour rejoindre l'opacité qui est notre propre. Mais c'est encore trop peu dire : c'est un propre qui permet de s'approprier ce dont nous sommes séparés : ce monde et son temps.

Cet étranger, nous l'avons vu flotter dans tant de rêves, ceux où nous savions voler, ceux où nous nous déplacions dans l'air sans le moindre effort. Rien de plus véridique que ce genre de rêves, qui sont sans doute à peu près les seuls à être archétypaux pour le commun des mortels. Aussi archétypaux que celui d'avoir tué et enterré un frère, un autre soi-même, de s'être séparé d'un jumeau qui n'est plus qu'une bulle, en effet, soufflée par la première expiration. Dans les bandes dessinées, les héros parlent dans des bulles, et cette figuration montre une extériorité de la pensée au corps. On prend connaissance de la pensée, sans voir que la bulle elle-même est l'entité nécessaire à son expression, toujours déjà prise dans la réflexivité. Au fond, ces échanges entre bulles expriment une vérité divisée que la parole occulte : ils montrent la transparence du lieu d'adresse qui nous reflète. Sur les dessins, les bulles se croisent et s'éclatent l'une l'autre, laissant les corps à distance, gesticulant à peine, très ralentis, ou plutôt immobiles par rapport à la vitesse de la pensée. En fait, le corps doit reconstituer son animation à partir de la pensée extérieure de la bulle, qui la tire par des ficelles comme une marionnette. Nous sommes à la merci de cette entité transparente, à laquelle nous obéissons sans la voir, comme si nous étions libres et autonomes. "Connais-toi, toimême! » Connais ta bulle et tu pétilleras, tu t'éclateras. L'analyse est une succession d'éclats de bulles, qui laissent un instant libéré, face à rien, pour aussitôt nous reprendre, ou plutôt être repris dans la distance à un nous-mêmes en bulles qui nous machinent. On croirait volontiers que la transparence est lumineuse mais la lumière, c'est déjà quelque chose qui traverse l'éther. En fait, nous ne percevons jamais notre propre transparence: nous parlons à notre bulle comme si nous nous parlions, alors que nous nous adressons à un corps perdu, séparé, à l'abri, en deçà de la luminosité. Nous ne nous en rendons jamais compte, sauf si, un jour d'hallucinose, notre propre 
bulle prend le dessus et nous adresse la parole, ou si nous la voyons hallucinée : un visage, des yeux, rendu méconnaissable par son exil.

Souvent, nous parlons tout seuls dans une bulle invisible au-dessus de nous. Souvent? Presque tout le temps en réalité! C'est seulement à de rares instants que nous adressons la parole à quelqu'un, si possible à quelqu'un qui lui ressemble, qui ressemble à ce jumeau devenu transparent, notre ange gardien, celui du désir d'origine. Quelque part en avant de nous, translucide et face à face, se tient l'idéal devant lequel nous nous justifions. Sa présence est certaine, lui que nul ne voit jamais, parmi ceux qui ont traversé l'espace de l'expiration. Personne, ou presque, parce que c'est lui que l'halluciné voit brusquement apparaître venant de l'Autre monde, lui donnant les ordres auxquels il aurait dû obéir, plutôt que de crier.

Nous ne le voyons jamais, mais nous pressentons sa présence, car l'heure de l'expiration a-t-elle jamais complètement fini de sonner? Ne devons-nous pas de nouveau inspirer à chaque instant ce qui va nous pousser encore, sinon à crier, du moins à penser ou à parler? Oui, nous n'arrêtons jamais de penser et de parler, aussi bien pour nous tenir à distance de ce nous-mêmes halluciné et provisoirement transparent, que pour tenter, dans le même geste, de nous rejoindre, de nous réconcilier. Dans la même temporalité alternée, Thomas l'obscur ${ }^{6}$ de Blanchot, hallucine et délire. Son délire, c'est son obscurité retrouvée contre l'éclair de l'hallucination. Celui qui subit la violence d'une hallucination tente ensuite de la justifier et de l'intégrer en construisant un délire. C'est à la fin d'Aminadab ${ }^{7}$ au moment où toute parole lui semble retirée que Thomas peut dire : «Maintenant, je parle ». La parole tire sa force de ce qu'elle dissimule, et cette force se laisse pressentir dans le silence, qui seul se souvient de la transparence, vide d'intériorité, à la fois origine et ce qui n'a pas de fin. La même transparence nous accompagne et balbutie dans chaque parole : elle roule comme un jeu de quilles, renversée à chaque mot, et renaissante dès que le silence revient. [...] « Il n'est pas une existence qui, dans la seule affirmation du Je parle, reçoive la promesse menaçante de sa propre disparition, de sa future apparition ${ }^{8}$ ».

Donc, il faut croire que nous devions pour toujours rester séparés, en exil de la transparence, même à l'heure d'une expiration définitive, qui laissera notre âme translucide dans un éternel suspens, comme les hommes en ont toujours eu l'intuition. L'expiration ne restera-t-elle pas pour toujours séparée de l'inspiration, comme l'âme l'est du corps ? Pourtant, il arrive que le bref instant d'une réconciliation sonne l'heure d'une retrouvaille du transparent et de l'obscur: d'un corps hallucinatoire et d'un corps pulsionnel pour le dire autrement. À l'heure de l'Aura, un monde irisé comme une bulle de savon brille un instant et fait miroiter des correspondances multiples, joyeuses, où tout devient clair. À l'heure de la crise d'asthme, ou à l'heure de la crise d'épilepsie (qui succèdent souvent à une aura), les spasmes des convulsions cognent en quelque sorte le corps à son double - ou bien dans l'asthme, l'inspiration se marie à l'expiration. À l'heure de l'orgasme, le corps se divise sous le coup de son étrangeté à lui-même : c'est ce qui le fait crier comme au premier jour.

11 L'intimité de notre séparation n'est pas en exil de quelque chose: nous avons un certain rapport - tremblant - à cette "extimité", pour reprendre l'expression de Lacan. Bien plus, cela se voit! Il existe de «bonnes » et de «mauvaises photos », celles dont le sujet ne se reconnaît pas sur la pellicule, qui n'a su fixer qu'une partie de la bascule temporelle, et non le point d'équilibre de la présence du sujet, entre le diaphane de son corps passé, et l'hallucinatoire du désir qui le porte. Pour parler de la 
photo, le terme de «pellicule» est presque le mot juste, pas tout à fait exact, car il donne l'idée d'un feuilletage qui pourrait être multiple alors qu'il s'agit de la localisation d'une contradiction temporelle. Être "pris» en photo (en sandwich, en somme) peut provoquer un petit instant d'angoisse, celle de voir ensuite sur le papier un déséquilibre trop appuyé, ou plutôt un refus du déséquilibre, un visage figé en dehors de sa distemporalité, déjà mort, en somme. Lorsque Nadar prit pour la première fois Balzac en photo, l'écrivain mal à l'aise chercha l'explication de son appréhension. Selon lui, "Chaque corps dans la nature se trouve composé de séries de spectres, en couches superposées à l'infini, foliacées en pellicules infinitésimales... chaque opération daguerrienne venait donc surprendre, détachait et retenait en se l'appliquant une des couches du corps objecté ${ }^{\text {}}$ P Pourtant, s'agit-il de tous les corps, ou seulement de celui de l'homme ? Il est probablement impossible de rater la photo d'un animal. Un visage humain, en revanche, est pris dans un constant tremblement qui peut le figer dans ce que Nadar appelle si justement son "objectité ${ }^{10}$ ", terme bien différent de l'« objectivité », toujours indiscutable d'une photo : comme l'écrit Didi-Huberman, ce serait alors « comme un effet mortifère de l'aura: la dévoration diaphane, par l'image, $\mathrm{du}$ folio des $\operatorname{corps}^{11} »$. L'instabilité qui résulte de la division subjective n'est pas stabilisée par l'objectivité du monde, qui tremble lui aussi sous le coup d'un corps qu'il reflète, en constante perte de vitesse. C'est ce qui est sensible sur n'importe quel tableau, ou même n'importe quelle photo : l'imminence de la chute dans l'avant ou d'un évanouissement dans l'après. C'est la façon de se raccrocher à un bord qui en fait le style, la façon d'attraper un petit détail pour surnager. Ce détail différentiel - en réalité capté par le style d'une subjectivité - n'est-il pas ce qui fait le propre de la perception telle que Leibniz la définit, par exemple. Et aussi telle que Freud la conçoit. La subjectivation salvatrice ne surgira pas dans la cure d'une explication, ou d'une théorie, mais d'un petit détail mémorisé au moment de la chute. L'inconscient est dans les détails.

On peut supposer que Balzac eut cette inspiration des spectres à cause de l'invention récente de la spectroscopie faite par Fraunhofer dans sa Théorie des Halos. Cet opticien génial décomposa le premier la lumière «blanche » en cinq cent soixante seize raies. On le suppose d'autant plus facilement que le héros du Chef d'œuvre inconnu se dénomme Frenhofer ${ }^{12}$ ! Mais le mot "spectre", introduit en optique, n'est-il pas étrange? Car avant d'être appliqué à la lumière, il désigne d'abord ce qui hante le visible, à commencer par le Mort (notre transparence première, si originelle qu'elle est ensuite rapportée à l'Ancêtre de la lignée, qui est, en ce sens, un sauveur : il rédime la transparence). Et, brusquement, la physique s'en est emparé pour décomposer notre pure lumière : le Spectre qui nous hante est désormais tenu à distance derrière la grille des 576 raies. Il est en quelque sorte repoussé justement jusqu'aux étoiles, puisque Fraunhofer a su établir une spectrographie des étoiles grâce à son optique. Au fond, cette invention, qui a inspiré le Chef d'œuvre inconnu et son issue fatale - par la destruction de l'œuvre et le suicide de l'artiste-, pourrait aussi à bon droit être considérée comme le premier meurtre scientifique de Dieu. Le Spectre, c'est-à-dire l'ancêtre mort a été pour la première fois numérisé par l'optique. Et la lumière - seule à dénoter la présence divine dans l'iconographie monothéiste - se décompose soudain en un chiffrage différentiel. L'artiste qui rate le moment de vacillation, et recouvre l'idéal futur par le mur de la transparence passée, ne pourra certes plus trouver son salut en portant un cierge à l'église. 
$13 \mathrm{Au}$ fond, les sensations présentent la réflexion du pli (figure du tremblement sur place). Et l'on pourrait croire que le saccage des pulsions, qui extériorisent le corps à luimême, s'embourbe et s'amortit dans les perceptions. Le pli pulsionnel est-il plus viable que la transparence d'origine? Mais, non ! Un infini plié devient méchant : son pli forcé en programme un autre, ou plutôt le corps plié cherche à se déplier, ressort qui fait sa structure cannibale intime. Ce corps n'est pas seulement pendu à l'extérieur, il s'est opacifié selon des zones érogènes, des significations locales finies d'un manque à être infini. Ce sont des lieux de belligérance, une guerre qui se déroule dans l'obscurité d'un corps devenu opaque à lui-même et qui se souvient à peine de sa pureté d'origine, sinon en rêve. C'est le lieu du sacré (sacer), il est interdit de l'ouvrir, de l'autopsier, de l'opérer : il incarne la maison de Dieu, est-il écrit dans le Livre, ce qui affirme d'une autre façon qu'il ne s'appartient pas. Le pli des pulsions cherche obstinément à se déplier, bien qu'il ne puisse le faire qu'à partir de ce qu'il est déjà devenu, à partir de ce point où la signification infinie de la matière l'a forcé à se plier. L'âme de la matière, c'est son pli à venir. Mais, du coup, la matière s'est perdue dans ce repliement. Elle cherche à se posséder en dehors de son être impossible, dans le possible de l'amour, si l'on appelle ainsi la discordance du rêve avec ce qu'il cherche à retrouver. C'est le second pli - sexuel - qui opacifie le corps une dernière fois contre la poussée des pulsions et grâce à elle. Désormais, le corps n'est plus qu'une fonction du désir, dont les variables déplacent l'érogénéité au hasard d'accidents oniriques et de rencontres fortuites. Par la voie de ces hasards, la machine se déclenche et cherche à reproduire ce qu'elle n'a jamais été. Double pliage du refoulement, pris dans les rythmicités d'un désir obsédé par une parade à la transparence renouvelée par l'amour. L'érotisme est son inflexion idéale, toujours déplacée dans la virtualité qui l'enveloppe. L'inflexion rejetée, l'âme du mouvement, c'est le sujet qui se débat avec sa matière d'origine, et pense au sexe comme à ce qui lui permettrait de se rejoindre. Rien ne l'effraie autant, donc. Il ne va pas se regarder en face, tout de même!

Dans l'amour, nous nous amincissons au point de redevenir transparents - ou presque ! Car quelque chose nous fait toujours nous ressaisir en dernière limite, juste avant le retournement complet, juste avant la chute mélancolique. Post coïtus animal semper triste : c'est l'heure où nous entrevoyons le corps animal d'origine qui pend au dehors. Un dicton prévient celui qui se marie qu'il va "se mettre la corde au cou». Dans leurs grandes crises, entre tous les moyens d'en finir, les mélancoliques préfèrent la pendaison, qui installe un rapport à l'espace plus étendu que se jeter par la fenêtre, tentation commune - plus petit commun dénominateur des amants de la transparence. "Tu me manques, je suis perdu sans toi, ma rambarde visible, dans ton œil ouvert où je me vois ». Pour le mélancolique, la corde isole de son corps animal la tête, porteuse de ce regard, des yeux au milieu du visage, lieu d'érubescence de toutes les hontes, quartier général des pulsions, qui annoncent épisodiquement au monde la faute irrémédiable et sans explication d'avoir renié la transparence. Un pendu ne crie pas. Il parait que les hommes sont alors en érection, et, au Moyen Âge, certains croyaient que la mandragore poussait là où tombait leur semence. Dans l'ordinaire de l'amour partagé, la perte s'aperçoit malaisément. Elle s'énonce, sans doute ( $\mathrm{Tu}$ me manques »...), mais elle se rattrape toujours aux branches, la moindre d'entre elles n'étant pas celle de l'érotisme - propre à provoquer l'érection qui, en somme, précède en ce cas la pendaison - non sans la pointe mélancolique de son soulagement. 
La folie de l'érotisme est-elle ainsi close sur elle-même - toujours au bord du drame ? Non, car il existe un geste que fait presque machinalement le mélancolique de la transparence, un geste qui le protège jusqu'à un certain point, en isolant pour luimême le moment de la perte : c'est l'acte de représenter, celui de dessiner, de peindre, de sculpter, de chanter... En ce sens, un tableau se pend. Le peintre d'abord, l'esthète ensuite (toi ou moi, n'importe qui) se voit ensuite dehors, encadré tranquillement, rivé à sa ficelle. Partout où des hommes habitent, on trouvera des gravures, des tableaux, des reproductions, ou bien de simples graffiti de corps, des photos de femmes découpées dans les magazines, ou, aujourd'hui les grandes affiches publicitaires, presque toutes dédiées à la gloire de la féminité, de ce qu'elle dérobe à chacun. C'est une façon de nous river notre clou dehors, de conjurer notre partage. Dans La peinture incarnée, Georges Didi-Huberman écrit: «l'enjeu en est au fond la constitution d'un sujet de l'énonciation, donc celle du sujet tout $\operatorname{court}^{13}{ }^{13}$. C'est un sujet qui vit dans le retranchement de son rêve, un sujet retranché de ce qu'il expose sous le jour de l'art, qu'il a produit en dépit de lui-même... « contre le gré de l'homme » écrit Balzac dans Le chef-d'œuvre inconnu ${ }^{14}$.

16 On pourrait dire qu'il s'agit d'un troisième pli, après celui de la pulsion et celui de l'érotisme (ce serait si l'on veut le pli de la sublimation). Mais, peut-on l'appeler "troisième ", puisqu'il doit faire l'ostension conjointe des deux autres - de la matière pulsionnelle prise dans son histoire: de l'infini plié entre passé et futur, entre transparence et opacité - deux en un troisième, en somme. Un tableau retarde-t-il, ou bien au contraire est-il toujours en avance, eu égard à l'hallucination de celui qui le peint? Ce n'est pas tant qu'il va manquer son achèvement du fait de ce décalage. Son achèvement est dans ce manque lui-même : c'est ce qu'on aimerait voir enfin, qui nous calmerait. Sinon, le tableau ne doit-il pas être détruit, c'est-à-dire ne faut-il pas le retourner dans les limbes de la transparence, où il va continuer d'animer les hallucinations sur place, qui pelliculent l'infinité des perceptions, qui leur donnent leur dimension infinie, creusée à même le perçu. Dans les musées, il est interdit de toucher les tableaux, et, très souvent, les enfants enfreignent cette consigne, lorsqu'un tableau leur plaît. C'est que, plus que les objets ordinaires, les tableaux sont nimbés d'une Aura hallucinatoire qui pousse à les toucher pour lever le doute que cette Aura fait naître. «Le doute et le toucher doivent être réunis » écrit H. Legrand du Saulle ${ }^{15}$ dans son article « Folie du doute (avec délire du toucher)».

Voir sans toucher est une épreuve, puisque la vision prend son élan à partir d'un irréel. C'est peut-être pourquoi les regards font le plus souvent semblant de ne pas se voir. On se croise comme si de rien n'était, sans se voir. Si l'on se regarde, c'est trop tard : non pas qu'il faudrait aussitôt se toucher pour exorciser l'hallucinatoire, mais à coup sûr, il faudrait parler. La vision déclenche une compulsion à parler plutôt qu'à toucher, et à vrai dire, les regards sont les rails de la parole. Un enfant que sa mère ne regarde pas parlera plus tard. Ses mots ne sont pas aspirés hors de lui par le trou du regard, dont le centre noir montre sans égard notre transparence première. Voir le regard impulse le moment où la voix doit prendre forme, parce qu'elle doit absolument écranter cette angoisse de l'origine selon un sens qui tente de donner une signification locale à la captation tactile des corps, à leur attachement dubitatif.

Car que se passera-t-il, si les corps se touchent - au moins la première fois ? Ne faut-il pas franchir une sorte d'obstacle, celui de la pudeur, du refus, comme s'il fallait retourner le pli du premier jour. Le sang de l'érubescence honteuse, c'est le contraire 
de la transparence, on l'a dit. Le visage, porté au rouge résiste à sa disparition dans l'absence de couleur, une pâleur mortelle qui rebondit dans un trop plein de présence. Lorsque Pygmalion ramène à la vie la statue d'ivoire qu'il a sculptée, la rougeur est le premier signe de cette naissance sous les caresses de son sculpteur. Sensit et eribuit, écrit Ovide : "elle sent ses caresses et rougit » car dans le même instant, le regard la fait vivre : "Elle a vu en même temps le ciel et son amant. » Voir le ciel, s'éveiller à l'érotisme en découvrant un visage penché sur le visage qui s'éveille donne l'idée d'une simultanéité entre l'échange de regards et la profondeur du ciel. Mais en réalité, l'érotisme, les caresses qui ont précédé l'éveil ne sont-ils pas la condition plus générale des perceptions? Sans elles, le monde demeure aussi plat que la statue reste de marbre. La profondeur de champ grâce à laquelle les sensations se distinguent les unes des autres réclame un moment de recul creusant sa profondeur. Eribuit : c'est la pudeur qui le creuse. Grâce au corps qui - en naissant- rougit et se refuse, naît la tridimensionnalité. La statue perd sa blancheur en même temps que le monde gagne le différentiel de ses opacités.

Pourquoi une femme, l'idée d'une femme, la Femme, est-elle si constamment l'obsession de l'œuvre, en tout cas dans les arts visuels comme la sculpture ou surtout la peinture? On pourrait dire que les femmes plutôt que les hommes symbolisent le manque qui qualifie en propre l'espèce humaine. Mais encore faudrait-il préciser ce que l'on entend par "symboliser ", car c'est plutôt du refus du manque dont il est question : le geste de pudeur, la honte, la retenue, le refus : tous aveux d'un désir qui demande d'abord l'aveu d'un autre désir : d'un manque au-delà du corps en somme, c'est-à-dire d'un corps homogène à sa transparence d'origine. L'obsession du féminin concerne ce qui d'elle ne se voit pas, au-delà de ce qu'elle refuse en un geste, en une posture de retrait. Car si la femme était symbole du manque, elle devrait être aussi celui de la consommation. Or, ce sont plutôt les hommes qui affichent un tel appétit, et donnent les signes les plus débordants d'un manque impénitent. Comme l'écrit Balzac, dans sa Théorie de la démarche: «En marchant, les femmes peuvent tout montrer, mais ne rien laisser voir ». L'obsession est du côté du « rien », dira-t-on sans doute pour satisfaire la fonction du symbole. Mais comment ce «rien» pourrait-il se laisser deviner, sinon dans une démarche justement, une pudeur, une retenue, un refus? En ce retrait, ce n'est pas l'homme que la femme refuse, mais son propre féminin. Le refus forge l'armature actuelle de la transparence, celle d'une beauté à faire peur devant laquelle les hommes reculent, comme ils reculent devant les femmes les plus belles, peur qu'ils ne peuvent outrepasser sans quelque violence, infligée à cette femme-là ou à euxmêmes, une violence de prédateur ou d'alcoolique. C'est celle d'Orphée qui se retourne pour voir Eurydice et fait disparaître dans son geste un corps entièrement devenu lieu de l'absence. Dans le tonnerre de l'élan sexuel gronde l'effet lointain de l'éclair premier de la transparence. On ignore ce qui pétrifie sur place la femme ou l'homme, ou seulement l'un des deux, ou les deux simultanément. Le chef d'œuvre inconnu de Balzac propose un tel scénario, le peintre passant une bonne part de sa vie à masquer, à recouvrir le tableau d'une beauté première, pour se suicider enfin. Il faut tout un scénario, une démarche, une histoire pour actualiser ce rapport à un corps d'origine immobile. Il faut le dédoubler sous les espèces du féminin, puis approcher ce féminin et dans cette approche, sentir l'élasticité d'une résistance, comme si l'air s'épaississait au fur et à mesure que la distance se réduit, jusqu'à former un mur infranchissable, qui empêche de totalement l'étreindre. Enfin, prendre son pinceau, et peindre le mur luimême, essayer de rendre l'idée de ce mur diaphane. C'est dire que le désir sexuel n'est 
qu'un temps de cette approche, une mise en pli au troisième degré, une opacification provisoire et duplice de la transparence. La femme qui incarne le rapport au manque fait renaitre le rapport au double. Elle le dislocalise, le disloque et se disloque aussi le temps du refus, celui de montrer ce qu'elle n'est pas. C'est ce qu'elle n'est pas qui compte en terme de désir, et c'est pourquoi la représentation, le rêve, le fantasme, le tableau, la sculpture montrent la vérité de ce féminin arrêté qui règne à mille lieux de n'importe quelle femme vivante. En somme, cette femme existe d'abord en rêve. Elle domine n'importe laquelle de ses incarnations et rien ne saurait la réduire, toujours déjà œuvre d'art, moteur secret de l'art, peut-être. Orphée chante dans l'espace de ses retrouvailles, puis de sa perte avant d'être dévoré par les Bacchantes. Son destin présente une symétrie inversée saisissante avec celui du sculpteur Pygmalion - comme le fait remarquer Didi-Huberman ${ }^{16}$; de la rechute aux enfers ou de l'ivoire qui se ranime. Si l'on considère le fléau de cette balance dans sa bascule immobile, aucun de ses plateaux ne porte la vérité. «Les dieux ont caché ce qui fait vivre les hommes ». C'est tout du moins ainsi qu'Hésiode regarde la transparence dans Les Travaux et les Jours $^{17}$. La disjonction n'apparaît pas, sinon comme une cause d'après-coup, pouvant certes piquer l'érotisme, mais en lui annonçant à l'avance le programme de son relatif échec.

\section{BIBLIOGRAPHIE}

Aristote, De Anima, II, 7, 418, Paris, Vrin, 1972.

Balzac Honoré de, «Le chef d'œuvre inconnu », in Études philosophiques, Delloye et Lecoue, t. XVII, 1837.

Blanchot Maurice, Aminadab [1942], Paris, Gallimard (L'imaginaire), 2004.

Blanchot Maurice, Thomas l'obscur [1941], Paris, Gallimard, 2005.

Deleuze Gilles, Le Pli, Paris, Minuit, 1988.

Didi-Huberman Georges , La peinture incarnée, Paris, Minuit, 1985.

Foucault Michel, La pensée du dehors, Fontfroide-le-Haut, Fata Morgana, 1986.

Hésiode, Les Travaux et les Jours, Paris, Belles Lettres, 1928.

\section{NOTES}

1. M. Foucault, La pensée du dehors, Fontfroide-le-Haut, Fata Morgana, 1986, p. 60.

2. Aristote, De Anima, II, 7, 418, Paris, Vrin, 1972, p. 105.

3. G. Didi-Huberman, La peinture incarnée, Paris, Minuit, 1985, p. 31.

4. Aristote, De Anima, III, 435a, p. 215.

5. G. Deleuze, Le Pli, Paris, Minuit, p. 113.

6. M. Blanchot, Thomas l'obscur [1941], Paris, Gallimard, 2005. 
7. M. Blanchot, Aminadab [1942], Paris, Gallimard (L'imaginaire), 2004.

8. M. Foucault, La pensée du dehors, p. 61.

9. G. F. Nadar, 1900, Quand j'étais photographe, Paris, Flammarion, p. 5-6.

10. Cette « objectité » est en réalité une position subjective de celui qui est pris sous le feu de ce qui est là aussi appelé si justement « l'objectif » de l'appareil de photo.

11. G. Didi-Huberman, La peinture incarnée, p. 35.

12. Ibid., p. 35.

13. Ibid., p. 14.

14. H. Balzac, «Le chef d'œuvre inconnu », in Études philosophiques, Delloye et Lecoue, 1837, t. XVII, p. 7.

15. H. Legrand du Saulle, «Folie du doute (avec délire du toucher)» 1875, p. 6. Cité par G. DidiHuberman, La peinture incarnée, p. 16.

16. Didi-Huberman, La peinture incarnée, p. 117.

17. Hésiode, Les Travaux et les Jours, Paris, Belles Lettres, 1928, vers 42, p. 87.

\section{AUTEUR}

\section{GÉRARD POMMIER}

Psychiatre, psychanalyste, Gérard Pommier est professeur de psychopathologie à l'université de Strasbourg, membre de Espace analytique, directeur de la revue La Clinique lacanienne et cofondateur de la Fondation européenne pour la psychanalyse. Il a notamment publié Comment les neurosciences démontrent la psychanalyse (Flammarion, 2004) et Les Corps angéliques de la postmodernité (Calmann-Lévy, 2002). 